\title{
VARIÁVEIS DEMOGRÁFICAS E O COMPORTAMENTO DO CONSUMIDOR DE ÁGUA: ESTUDO DE UMA POPULAÇÃO UNIVERSITÁRIA
}

\author{
DEMOGRAPHIC VARIABLES AND THE BEHAVIOR OF THE WATER CONSUMER: \\ STUDY OF A UNIVERSITY POPULATION
}

\section{VARIABLES DEMOGRÁFICAS Y EL COMPORTAMIENTO DEL CONSUMIDOR DE AGUA: ESTUDIO DE UNA POBLACIÓN UNIVERSITARIA}

\begin{abstract}
Andréia de Fátima Hoelzle Martins, Programa de PósGraduação em

Administração Universidade Federal de Viçosa, Viçosa, MG, Brasil

Daiane Medeiros Roque, Programa de PósGraduação em

Administração Universidade Federal de Viçosa, Viçosa, MG, Brasil

Afonso Augusto de Freitas de Carvalho Lima, Programa de PósGraduação em

Administração Universidade Federal de Viçosa, Viçosa, MG, Brasil
\end{abstract}

Marco Aurélio Marques Ferreira, Programa de PósGraduação em

Administração Universidade Federal de Viçosa, Viçosa, MG, Brasil

Artigo Submetido em 16.08.2017. Ajustado e Aprovado em 10.05.2018. Avaliado pelo processo de double blind review

e-mail para contato: hmartins.andreia@gmail.co

\author{
RESUMO
}

O consumo sustentável tem sido tema recorrente na sociedade, tendo em vista as consequências do uso indiscriminado dos recursos naturais. Água, bem indispensável à vida, é parte destes recursos e também é utilizada de forma negligente, agravando as situações de restrição hídrica, vivenciadas no Brasil, desde 2014, especificamente nas regiões Sudeste e Nordeste. Neste sentido, conhecer o perfil e o comportamento do consumidor de água é relevante para a elaboração de políticas indutoras ao comportamento sustentável. Assim, o objetivo do estudo foi conhecer o comportamento do consumidor de água de um público universitário, relacionando-o com os aspectos demográficos dessa população. Para tanto, utilizou-se de dados quantitativos e regressão linear para coleta e análise de dados, respectivamente. Como resultado, o índice do comportamento declarado do consumidor de água demonstrou que os respondentes são propícios ao consumo sustentável, e que os consumidores do gênero feminino, com alta renda e escolaridade são mais predispostos a terem comportamentos de consumo sustentáveis.

Palavras-chave: Comportamento do consumidor; Consumo sustentável; Perfil demográfico dos consumidores.

\section{ABSTRACT}

Considering the indiscriminate use of natural resources, sustainable consumption has been a recurrent concern of governments and societies around the world. Water, essential to life also used of a negligence way, behavior that aggravates in water crises situation, such as experienced by Brazil, specially at southeast and northeast regions. In this sense, understand water consumer behavior and their demographic characteristics contributes to public administration, been important to elaborate public policies to encourage water sustainable behaviours. Thus, our purpose was understood water consumer behavior of a university population (UFV), testing their relationship with their demographic characteristics. Quantitative data and Linear Regression were used to data collect and data analyse, respectively. Based on the results, we can conclude that water consumer behavior present medium or high levels to sustainable practices involving water consumption, and that consumers of female gender, high levels of rent and education are more prone to sustainable behaviours.

Key-words: Consumer Behavior, Sustainable consumption, consumer demographic profile 


\section{QREUNIR}

\section{RESUMEN}

El consumo sostenible ha sido tema recurrente en gobiernos de todo el mundo, considerando las consecuencias del uso indiscriminado de los recursos naturales. El agua, bien indispensable para la vida, es parte de estos recursos que también es utilizada de forma negligente, este uso agrava situaciones de restricción hídrica, como las vivenciadas en Brasil, desde 2014, específicamente en las regiones Sudeste y Nordeste del país. En este sentido, conocer el perfil y el comportamiento del consumidor de agua es relevante para la elaboración de políticas inductoras del comportamiento sostenible. Así, el objetivo del estudio fue conocer y comprender el comportamiento del consumidor de agua de un público universitario, relacionándolo con los aspectos demográficos da populación estudiada. Para ello, se utilizaron datos cuantitativos y regresión lineal para recolección y análisis de datos, respectivamente. Como resultado, el índice del comportamiento declarado del consumidor de agua ha demostrado que los investigados son propensos al consumo sostenible, y que los consumidores del género femenino, con alta renta y escolaridad, están más predispuestos a tener comportamientos sostenibles.

Palabras clave: Comportamiento del consumidor; Consumo sostenible; Perfil demográfico de los consumidores.

\section{INTRODUÇÃO}

O consumo sustentável ${ }^{1}$ tem sido um assunto frequente das pesquisas e agendas de governo de diversos países (RIBEIRO e VEIGA, 2011). Tal tema tornou-se relevante a partir da percepção sobre as consequências dos impactos ambientais, sociais e econômicos do uso indiscriminado dos recursos naturais.

A água é um recurso natural essencial para a nossa alimentação e higiene, sendo também indispensável para o equilíbrio ambiental e manutenção da economia (VACCARI, 2014). Após a Constituição de 1988, atribuiu-se a União à responsabilidade de preservação e controle dos recursos hídricos de modo a promover seu uso sustentável (BRASIL, 1988).

De modo a cumprir a legislação, o governo elabora políticas ambientais, que podem ser regulatórias (legislações), estruturadoras (intervenção direta em defesa do meio natural) e indutoras, que têm por objetivo influenciar o comportamento dos cidadãos e dos grupos sociais (CUNHA e COELHO, 2003). Considerando que a promoção do uso sustentável da água é encargo do Estado, o conhecimento das variáveis que interferem na adoção do consumo sustentável de água pode ser relevante para as políticas indutoras. Além disso, contribuem para a elaboração de políticas amplas, capazes de diferenciar seus públicos e atuar diante deles de forma diferenciada.

Neste sentido, diversos autores apontam que as variáveis sociodemográficas (SOLOMON, 2011; KOLLMUS e ANGYEMAN, 2002) e psicográficas (RIBEIRO e VEIGA, 2011; STRAUGHAN e ROBERTS, 1999) são relevantes para moldar a adoção ou não de determinado

\footnotetext{
${ }^{1}$ Entendeu-se consumo sustentável conforme a definição de Vaccari (2014) como o comportamento da sociedade, empresas e governos em relação a adoção de práticas ambientalmente corretas, de forma a garantir a sustentabilidade dos recursos naturais para as gerações futuras.
} 


\section{(9)REUNIR}

comportamento. Contudo, poucas pesquisas tiveram como foco a análise de variáveis que apresentavam relação com o consumo de água (GILG e BARR, 2006; RANDOLPH e TROY, 2008). E ainda, dentre os estudos, há pouca concordância entre os autores sobre quais variáveis realmente poderiam ser consideradas importantes (GILG e BARR, 2006).

A maioria das pesquisas que se dedicaram ao estudo consumo sustentável, abordando as variáveis demográficas e psicográficas, não incluíram o consumo de água (RIBEIRO e VEIGA, 2011; STRAUGHAN e ROBERTS, 1999; AZEVÊDO et al., 2010; GOMES, GORNI e DREHER, 2010). Quando abordado, este era entendido apenas na dimensão dos hábitos que envolviam a economia de água (VACCARI, 2014).

Diante dos ainda incipientes estudos sobre as variáveis que possivelmente influenciam no comportamento do consumidor de água, o objetivo deste estudo foi identificar se as variáveis demográficas implicam em diferentes comportamentos em relação ao consumo sustentável da água na população universitária da Universidade Federal de Viçosa (UFV).

Especificamente, propôs-se: Descrever os comportamentos declarados dos consumidores de água da Universidade Federal de Viçosa; identificar se há diferenças de comportamentos entre as variáveis demográficas, e quais estão mais relacionadas ao consumo sustentável de água.

Optou-se pela cidade de Viçosa (MG), devido ao contexto hídrico vivenciado pela cidade, que desde 2014 passa por uma crise hídrica devido à ausência de chuvas e crescimento da demanda $(G 1,2016)$. Tal crise implicou na necessidade de mudança de comportamento da população, que foi convidada pelo governo e demais instituições a economizar o recurso.

Quanto à população universitária, esta foi escolhida devido à característica deste público, sendo os professores e alunos atuais e futuros formadores de opinião (STRAUGHAN E ROBERTS, 1999). Autores como Bedante (2004); Franco (2012) e Grohmannet al. (2012) estudaram o comportamento do consumo consciente e utilizaram como amostra, populações universitárias.

\section{FUNDAMENTAÇÃO TEÓRICA}

Apresenta-se neste tópico as orientações teóricas que guiaram o entendimento do comportamento do consumidor de água e das variáveis demográficas relevantes para o comportamento sustentável, visando fundamentar o problema e objetivo proposto no estudo, bem como as escolhas metodológicas realizadas.

\subsection{Comportamento do Consumidor de Água}

Samara e Morsh (2005) definem o comportamento do consumidor como o conjunto de necessidades, desejos e motivações que envolvem o comportamento das pessoas. 0 comportamento do consumidor envolve também "as atitudes com que as pessoas se ocupam quando obtêm, consomem e dispõem de produtos e serviços" (BLACKWELL, MINIARD e ENGEL, 2009, p.6). 


\section{(9)REUNIR}

Entender com profundidade o consumidor é um desafio para o marketing, considerando que o comportamento humano é complexo e pode ser moldado por diversos fatores (CAPELINI, 2007). Alguns autores dedicaram-se à elaboração de modelos que pudessem explicar a tomada de decisão para determinados comportamentos. Kollmus e Angyeman (2002) a partir de uma revisão bibliográfica, e posteriormente Vaccari (2014) enfatizaram os principais modelos, dentre eles, modelos que visavam o comportamento sustentável, conforme demonstra o Quadro 1.

\begin{tabular}{|c|l|}
\hline Modelo & \multicolumn{1}{c|}{ Variáveis Consideradas } \\
\hline $\begin{array}{c}\text { Comportamento Ecológico (Fietkau } \\
\text { e Kessel, 1981apud Vaccari, 2014) }\end{array}$ & $\begin{array}{l}\text { Atitudes e valores ambientais; Possibilidades de agir de forma pró- } \\
\text { ambiental; Incentivos comportamentais; Feedback do } \\
\text { comportamento pró-ambiental e Conhecimento ambiental }\end{array}$ \\
\hline $\begin{array}{c}\text { Comportamento Ambiental (Hineset } \\
\text { al., 1986) }\end{array}$ & $\begin{array}{l}\text { Atitudes; Lócus de controle; Sentido de responsabilidade individual; } \\
\text { Conhecimento das questões ambientais; Conhecimentos de } \\
\text { estratégias de ação; Capacidade de agir e compromisso verbal; } \\
\text { Fatores Situacionais. }\end{array}$ \\
\hline $\begin{array}{c}\text { Modelo da Teoria Valor-Crença- } \\
\text { Norma de Sternet al(1999) }\end{array}$ & Valores, Crenças e Normas Pessoais. \\
\hline $\begin{array}{c}\text { Barreiras de Preocupações } \\
\text { Ambientais e Ações (Blake, 1999) }\end{array}$ & Individualidade, Responsabilidade, Praticidade. \\
\hline $\begin{array}{c}\text { Modelo de Comportamento Pró- } \\
\text { Ambiental (Kollmus e Angyeman, } \\
\text { 2002) }\end{array}$ & $\begin{array}{l}\text { Conhecimento, Sentimentos, Valores e Atitudes, Infraestrutura, } \\
\text { Fatores Políticos, Sociais e Culturais, Situação Econômica. }\end{array}$ \\
\hline $\begin{array}{c}\text { Atitude Ecologicamente Correta } \\
\text { (Leonidou, Leonidou e Kvasova, } \\
\text { 2010) }\end{array}$ & $\begin{array}{l}\text { Fatores antecedentes (cultural, político e ético), Fatores de atitudes } \\
\text { (interna e externa), Fatores comportamentais e resultados. }\end{array}$ \\
\hline \multicolumn{2}{|c|}{ Quadro 1- Modelos de Comportamento Sustentável } \\
\hline
\end{tabular}

Das variáveis consideradas no Quadro 1 foram selecionadas aquelasrelacionadas ao comportamento sustentável de água, tendo em vista que este é parte do comportamento pró-ambiental (WALL et al., 2004).Tendo em vista os modelos e autores já apresentados, percebe-se que estes muitas vezes apontam variáveis em comum que influenciam o comportamento ambiental.

Assim, dentre as variáveis selecionadas para representar o comportamento sustentável de água estão:Hábitos (TRIANDIS, 1977; KOLLLMUS e ANGYEMAN, 2002), Conhecimento (FIETKAU e KESSEL, 1981apud Vaccari, 2014; HINES et al., 1986; KOLLMUS e ANGYEMAN, 2002; VACCARI, 2014), Influência das Campanhas de Conscientização (KOLLMUS e ANGYEMAN, 2002), Influência Social(FISHBEIN E AJZEN, 1980), Motivação (KOLLMUS e ANGYEMAN, 2002), e Condições Naturais (THOGERSEN, 2005).

Entendeu-se Hábitos como as ações rotineiras em relação ao comportamento ambiental (KOLLMUS E ANGYEMAN, 2002). Já o conhecimento é entendido como uma das determinantes relevantes, tendo em vista que a partir dele pode-se tomar consciência ou responsabilidade pelos problemas ambientais (KOLLMUS e ANGYEMAN, 2002; VACCARI, 2014). 


\section{(9)REUNIR}

No que tange às campanhas, estas também exercem o papel de levar conhecimento aos consumidores sobre os problemas ambientais, enfatizando a importância da adoção de determinados comportamentos (KOLLMUS e ANGYEMAN, 2002). A influência socialpode ser entendida como parte do que Fietkau e Kessel (1981) apud Vaccari (2014) chamaram de "Incentivos para o comportamento pró-ambiental". A partir desta variável, entende-se que o comportamento ambiental pode ser incentivado por grupos sociais, como família, amigos, vizinhos, dentre outros.

Quanto à motivação, conforme exposto por Vaccari (2014) esta engloba os afetos, relação custo/benefício, bem como as preocupações morais e normativas. Por fim, julgou-se relevante considerar as Condições Naturaisque podem ser entendidas como parte dos fatores externos que influenciam no comportamento sustentável, como por exemplo, o clima (VACCARI, 2014). No caso do consumo sustentável do uso da água, este pode ser motivado ou restringido pela escassez ou acúmulo de chuvas, por exemplo.

Além destas variáveis, são relevantes para influenciar o comportamento sustentável as os fatores psicográficos (STRAUGHAN e ROBERTS,1999) e demográficos (KOLLMUS e ANGYEMAN, 2002; VACCARI, 2014).

\subsection{Variáveis Demográficas e Comportamento Sustentável}

Straughan e Roberts (1999) analisaram, em seu estudo a influência das variáveis demográficas e psicográficas no comportamento sustentável. Em relação às variáveis psicográficas, foram considerados variáveis que apresentaram forte correlação com o comportamento sustentável, dentre elas estão o altruísmo e as crenças ambientais, também conhecidas como eficácia do comportamento percebido (PCE). A escala proposta pelos autores foi validada por outros autores, como Afonso (2010) e Ribeiro e Veiga (2011).

Quando as variáveis demográficas, autores como Roberts (1996); Straughan e Roberts (1999); Getzner, Grabner e Kräuter (2004); Solomon (2011); Vaccari (2014) defendem que Gênero, Idade, Renda, Escolaridade e Raça e Etnicidade são fatores relevantes para compreender o comportamento.

É importante enfatizar que os estudos que consideram as variáveis demográficas podem apresentar muitas variações em seus resultados, havendo em todos os casos estudos que contrariam as expectativas teóricas (STRAUGHAN e ROBERTS, 1999), principalmente tratando-se do consumo de água, que ainda foi pouco explorado, a maioria dos estudos que tratam do consumo verde ou do comportamento sustentável como um todo. Assim, buscouse elencar as expectativas teóricas em relação às variáveis demográficas, quando correlacionadas com o comportamento sustentável.

A expectativa em relação ao gênero, é que o gênero feminino tende a ser mais propicio ao comportamento sustentável (STRAUGHAN e ROBERTS, 1999; STERNet al., 1999; DACIÊ et al. 2015; SANTOS e CONKE, 2014). Quanto à idade, autores defendem que as gerações mais novas tendem a apresentar mais comportamentos sustentáveis, tendo em vista, que desde pequenos possuem mais contato com os conhecimentos referentes aos problemas ambientais (VACCARI, 2014; STRAUGHAN e ROBERTS, 1999).

Sobre a renda, há ainda mais discrepâncias teóricas nesta variável (STRAUGHAN e ROBERTS, 1999), tal fato possivelmente deve-se aos diferentes comportamentos 


\section{(9)REUNIR}

sustentáveis analisados. Espera-se que as pessoas de maior renda tenham mais consciência ambiental, já que também possuem mais condições de arcarem com maiores custos e geralmente têm níveis mais elevados de escolaridade (NEWELL e GREEN, 1997; DACIÊ, 2015; RIBEIRO e VEIGA, 2011). Isto varia, por exemplo, no consumo de água ou energia, que quanto maior a casa, maior tende a ser o consumo (RANDOLPH e TROY, 2008).

Tratando-se da escolaridade, a sua relação com o comportamento sustentável tem sido bastante consistente. Espera-se que quanto maior o nível de escolaridade, mais propenso ao consumo sustentável esteja o indivíduo (STRAUGHAN e ROBERTS, 1999; RIBEIRO e VEIGA, 2011).

No que se refere àraça e a etnicidade, esta variável é encontrada em poucos estudos, mas enfatizada por estudiosos do comportamento do consumidor. Solomon (2011) entende que a raça e a etnicidade podem estar ligadas ao comportamento do consumidor tendo em vista que muitas vezes estas estão relacionadas à cultura e a determinados hábitos.

\section{METODOLOGIA}

Apresenta-se neste tópico o decorrer metodológico adotado, tais quais o processo de coleta e análise dos dados, bem como os participantes da pesquisa.

\subsection{Coleta de Dados e Participantes da Pesquisa}

Para conhecer o comportamento de consumo de água da população estudada foi elaborado um questionário composto por questões em formato de afirmativas, em que o respondente poderia escolher entre concordar totalmente a discordar totalmente, em uma escala tipo Likert de 11 pontos.

O questionário foi dividido em constructos que representam o comportamento sustentável, de acordo com aliteratura consultada, conforme apresentado no Quadro 2. A identificação na literatura de fatores que podem determinar o comportamento sustentável foi determinante para o desenvolvimento dos constructos, visto queestes foram a base para a elaboração do questionário e para a análise dos resultados.

\begin{tabular}{|c|l|}
\hline Constructos & \multicolumn{1}{|c|}{ Fonte } \\
\hline Hábitos & Kollmus e Angyeman (2002); Vaccari (2014) \\
\hline Conhecimento & $\begin{array}{l}\text { Fietkau e Kessel (1981 apudVaccari, 2014); Hineset al. (1986); Kollmus e Angyeman } \\
\text { (2002); Vaccari (2014) }\end{array}$ \\
\hline Campanhas & Kollmus e Angyeman (2002) \\
\hline Influência Social & $\begin{array}{l}\text { Vaccari (2014); Fishbein e Ajzen (1980); Triandis (1977); Fietkau e Kessel } \\
\text { (1981apudVaccari, 2014) }\end{array}$ \\
\hline Motivação & $\begin{array}{l}\text { Fietkau e Kessel (1981 apud Vaccari, 2014); Hinesetal (1986); Leonidou, Leonidou e } \\
\text { Kvasova (2010); Kollmus e Angyeman (2002); Vaccari (2014) }\end{array}$ \\
\hline Condições Naturais & Thogersen (2005) \\
\hline & \multicolumn{1}{|c|}{ Quadro 2- Constructos Elaborado pelos autores }
\end{tabular}

A coleta de dados foi realizada através de questionários aplicados online para a comunidade acadêmica da Universidade Federal de Viçosa (UFV), que autorizou a realização

61 


\section{(9REUNIR}

da pesquisa conforme os termos do comitê de ética. A universidade tem como sede a cidade de Viçosa, escolhida devido à crise hídrica vivenciada, desde 2014, conforme já exposto na introdução.

Obteve-se um total de 806 respostas, dentre os respondentes estão os professores, técnicos administrativos e alunos da UFV, posteriormente à Análise exploratória dos dados obteve-se um total de 719 observações válidas. A distribuição de frequência segue apresentada nas tabelas abaixo.

Tabela 1 - Frequência Gênero

\begin{tabular}{c|c|c|}
\hline Gênero & Feminino & Masculino \\
\hline$\%$ & 53 & 47 \\
\hline
\end{tabular}

Fonte: Resultados da pesquisa(2016).

Tabela 2 - Frequência Idade

\begin{tabular}{c|c|c|c}
\hline Idade & Geração Y & $\underline{\text { Geração X }}$ & Geração Baby Boombers \\
\hline$\%$ & 81,9 & 11,8 & 6,3 \\
\hline \multicolumn{4}{c}{ Fonte: Resultados da pesquisa (2016). } \\
Tabela 3 - Frequência Cor/Raça
\end{tabular}

\begin{tabular}{c|c|c|c|c|c}
\hline Cor/Raça & Branca & Parda & Negra & Indígena & Amarela \\
\hline$\%$ & 63,6 & 29,3 & 5,3 & 0,4 & 1,4 \\
\hline
\end{tabular}

Fonte: Resultados da pesquisa (2016)

Tabela 4 - Frequência Escolaridade

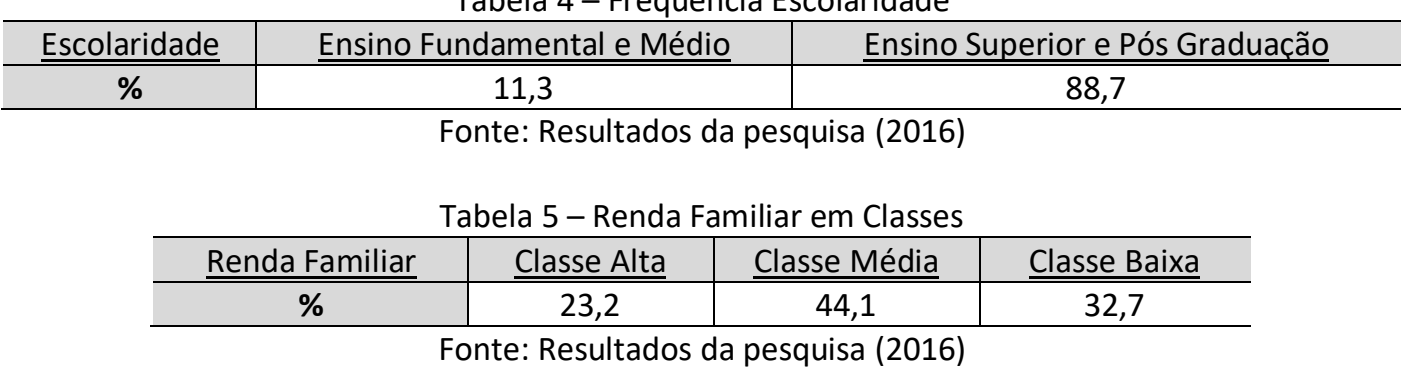

Com relação à Idade, para facilitar a apresentação e o agrupamento dos indivíduos, estes foram divididos em três grupos, a Geração X, a Geração Y e a Geração Baby Boombers. A geração Baby Boombers corresponde aos indivíduos nascidos após a segunda guerra mundial, já a geração $X$ contempla os indivíduos nascidos entre os anos 60 e 70 e a geração $Y$ nascidos após 1980.

A distribuição de frequência dos respondentes corresponde ao perfil da comunidade universitária, em que se concentram em jovens (Geração Y), pessoas de maior escolaridade (Ensino Superior e Pós-Graduação) e em sua maioria, de Classe Baixa e Média. Portanto, pode-se concluir que os dados representam bem a comunidade acadêmica da Universidade Federal de Viçosa.

\subsection{Hipótese Central}

Com base nos pressupostos definidos pela literatura, elaborou-se a seguinte hipótese: $\mathrm{H}_{0}=\mathrm{O}$ consumo sustentável de água não possui relação com variáveis 


\section{(9REUNIR}

demográficas; $\mathrm{H}_{1}=\mathrm{O}$ consumo sustentável de água possui relação com as variáveis demográficas.

\subsection{Expectativas Teóricas}

Tendo em vista que há constantes discordâncias entre a relação das variáveis demográficas e o comportamento sustentável, fez-se uma escolha dentre as expectativas mais defendidas dentre os autores, conforme apresenta o Quadro 3.

\begin{tabular}{|c|c|c|}
\hline Variável & Expectativa teórica & Fonte \\
\hline Gênero & $\begin{array}{l}\text { O gênero feminino tende a ser mais propício ao } \\
\text { comportamento sustentável. }\end{array}$ & $\begin{array}{l}\text { Eagly (1987) apudStraughan e } \\
\text { Roberts (1999); Stern et al. } \\
\text { (1999); Daciê et al. (2015); } \\
\text { Santos e Conke (2014) }\end{array}$ \\
\hline Idade & $\begin{array}{l}\text { As gerações mais novas tendem a apresentar } \\
\text { comportamentos mais sustentáveis, tendo em vista, que } \\
\text { desde pequenos possuem mais contato com os } \\
\text { conhecimentos referentes aos problemas ambientais. }\end{array}$ & $\begin{array}{l}\text { Vaccari (2014); Straughan e } \\
\text { Roberts (1999) }\end{array}$ \\
\hline Renda & $\begin{array}{l}\text { Espera-se que as pessoas de maior renda tenham mais } \\
\text { consciência ambiental, já que também possuem mais } \\
\text { condições de arcarem com maiores custos e geralmente } \\
\text { têm níveis mais elevados de escolaridade. }\end{array}$ & $\begin{array}{l}\text { Newell e Green (1997); Daciê } \\
\text { (2015); Ribeiro e Veiga (2011) }\end{array}$ \\
\hline Escolaridade & $\begin{array}{l}\text { Espera-se que quanto maior o nível de escolaridade, } \\
\text { mais propenso ao comportamento sustentável esteja o } \\
\text { indivíduo. }\end{array}$ & $\begin{array}{l}\text { Straughan e Roberts (1999); } \\
\text { Ribeiro e Veiga (2011) }\end{array}$ \\
\hline Raça & $\begin{array}{l}\text { Espera-se que haja diferenças entre as médias de } \\
\text { consumo sustentável de água para as diferentes } \\
\text { amostras de raça e a etnicidade, tendo em vista que } \\
\text { muitas vezes estas estão relacionadas à cultura. }\end{array}$ & Solomon (2011) \\
\hline
\end{tabular}

\section{Quadro 3 - Expectativas teóricas}

Fonte: Elaborado pelos autores com base na literatura

\subsection{Análise Dos Dados}

Os dados foram analisados com base em métodos quantitativos. Os métodos centrais utilizados para relacionar as variáveis demográficas ao comportamento foram os testes de associação (Correlação - Coeficiente de Spearman); Regressão logística e os Testes não paramétricos Kruskal-Walis e Mann-Whitney.

Inicialmente, de modo a validar os constructos elaborados calculou-se o Alfa de Cronbach de cada dimensão, com o intuito de verificar se as variáveis elaboradas são representativas, conforme orientado por Maroco e Garcia-Marques (2006). Posteriormente, recorreu-se à Análise Exploratória de Dados, que segundo Triola (2005) é a análise descritiva dos dados.

Tendo em vista a não normalidade dos dados, detectada pelo teste de KolmogorovSmirnov, recorreu-se a correlação de Spearman, utilizada para demonstrar as variáveis demográficas que apresentavam correlação com o comportamento sustentável de água.

Tomando como base os resultados da correlação, foi realizado o procedimento de regressão logística, que analisa um conjunto de variáveis explicativas $(X 1, X 2, \ldots, X n)$ em função de uma variável dependente (Y) (FÁVERO et al., 2009). Tendo em vista que as 


\section{(9REUNIR}

variáveis explicativas foram coletadas em forma de variáveis categóricas, estas foram transformadas em variáveis dummy, conforme indicado por Missio e Jacobi (2007), a exceção da variável idade, que foi coletada como contínua e assim foimantida. Do mesmo modo, transformou-se a variável dependente em uma dummy, tendo como ponto de cortea mediana, logo os valores acima da mediana foram considerados como "comportamentosmais sustentáveis" e valores abaixo da mediana como "comportamentosmenos sustentáveis".

Assim, a técnica permitiu a visualização das variáveis demográficas com maior poder para explicar o consumo sustentável. A opção pela regressão logística está associada à natureza dos dados coletados, que não apresentaram os preceitos da normalidade, além disso, esta é uma técnica robusta, que pode ser aplicada em várias situações (HAIR et al., 2009).

Entretanto, antes de realizar o modelo de regressão logística, recorreram-se aos testes de médias de modo a conhecer a relação entre as variáveis demográficas e o comportamento de consumo de água. Sabendo da não-normalidade dos dados, foram utilizados os testesMann-Whitney e Kruskal-Wallis. A hipótese nula é que não há relação entre a variável dependente e a variável demográfica testada.

Por se tratar de uma pesquisa com base no comportamento declarado dos participantes,os outliers não foram retirados da amostra, visto que conhecer a dispersão das variáveis dentro da distribuição é importante na compreensão do comportamento de consumo dos respondentes. Deste modo, optou-se apenas por retirar as observações com baixa qualidade, ou seja, as que apresentaram a mesma nota para todas as afirmativas.

\section{RESULTADOS E DISCUSSÕES}

Apresenta-se abaixo os resultados da pesquisa, em que é apresentado o comportamento do consumidor de água da UFV e posteriormente sua relação com as variáveis demográficas.

\subsection{Comportamento do Consumidor de Água na UFV}

Antes derelacionar as variáveis demográficas ao comportamento do consumidor, é importante conhecer o comportamento geral dos respondentes. Sabendo-se que todos os constructos que formam o comportamento do consumidor de água foram validados a partir do Alfa de Cronbach, este foi formado a partir da soma das respostas de cada constructo, em que o valor máximo do comportamento equivale a 220 pontos. Considerando que quanto mais alto for o valor do comportamento do consumidor de água, mais propício ele está ao uso sustentável da água este pode ser analisado com base na Tabela 6 .

Tabela 6 - Comportamento do consumidor de água

\begin{tabular}{l|c|c|c|c|c|c}
\hline \multirow{2}{*}{ Comportamento do consumidor de água } & $\underline{\text { Média }}$ & $\underline{\text { Moda* }}$ & $\underline{\text { Med. }}$ & $\underline{\text { Desv. Padrão }}$ & $\underline{\text { Min. }}$ & $\underline{\text { Máx. }}$ \\
\cline { 2 - 8 } & 162,87 & 158 & 167 & 30,134 & 15 & 217 \\
\hline
\end{tabular}

*Há vários valores modais, o menor valor foi demonstrado

Fonte: Resultados da Pesquisa (2016)

64 


\section{(9REUNIR}

Analisando os dados obtidos na tabela 6 vê-se que os valores encontrados para a média $(162,87)$, moda (158) e mediana (167) estão mais próximos ao final da escala (220), o que indica que a maior parte dos respondentes declaram adotarcomportamentossustentáveis em relação ao consumo de água. Ressalta-se a partir da análise da mediana que, 50\% dos valores estão entre 167 e 217, isto demonstra concordância com as afirmativas, contribuindo para o entendimento de que há propensão aoscomportamentos sustentáveis em relação à água na população de estudo.

Contudo, é importante destacar que há grande variabilidade dentre as repostas, sendo o valor mínimo equivalente a 15 e o Máximo a 217. Neste sentido, podemos observar que a variância é alta, demonstrado também pelo Desvio Padrão.

Tais resultados podem ser justificados devido à situação de restrição hídrica na cidade de Viçosa-MG, em que, desde 2014 os cidadãos são constantemente lembrados sobre a relevância de economizar água e utilizá-la de modo sustentável, o que possivelmente tem implicado na adoção de comportamentos sustentáveis no uso da água. $E$ ainda, é relevante destacar que ao lidar com dados do comportamento declarado, o respondente normalmente tende a passar uma imagem positiva de seus comportamentos, 0 que pode revelar o comportamento declarado mais positivo do que o comportamento real.

\subsection{Comportamento do Consumidor de Água e Variáveis Demográficas}

Com o intuito de refutar a hipótese Ho proposta, a qual acredita-se que o consumo sustentável de água não possui relação com variáveis demográficas, realizou-se a correlação de Spearman e a regressão logística para verificar a relação entre o comportamento do consumidor de água e as variáveis demográficas.

Tendo em vista que os dados não apresentam distribuição normal, recorreu-se ao coeficiente de Spearman, para isso, os dados referentes à escala somada de comportamento referente ao consumo sustentável de água foram transformados para uma escala de postos. A Tabela 7 apresenta os resultados das variáveis que apresentaram correlação significativa com a variável dependente.

Tabela 7 - Correlação de Spearman

\begin{tabular}{cccccc}
\hline & Gênero & Idade & Escolaridade & Cor/raça & $\underline{\text { Renda }}$ \\
\hline Correlação de Spearman & $-0,115^{* *}$ & $0,148^{* *}$ & $0,179^{* *}$ & $-0,093$ & $-0,026$ \\
\hline Sig. & 0,002 & 0,000 & 0,000 & 0,013 & 0,488 \\
\hline
\end{tabular}

**. A correlação é significativa no nível 0,01 (2 extremidades).

*. A correlação é significativa no nível 0,05 (2 extremidades). Fonte: Resultados da Pesquisa (2016)

Tendo em vista as variáveis indicadas, considera-se o Gênero, Idade, Raça e Escolaridade como variáveis de correlação significativas em relação ao comportamento do consumidor de água, sendo, portanto, relevantes para serem incluídas na primeira tentativa do modelo de regressão. Ainda de acordo com os resultados é possível constatar que as variáveis Gênero e Cor/Raçaapresenta uma relação inversa com o consumo sustentável, devido o sinal negativo apresentado, fato que leva a concluir que um dos gêneros e alguma 


\section{(9REUNIR}

raça contribuem negativamente com o consumo sustentável, sendo isto revelado com osTestes u de Mann Whitney e Kruskal Wallis.

A idade e a escolaridade contribuem positivamente com o consumo sustentável de água, ou seja, quando maior a idade e a escolaridade, mais inclinado está o indivíduo para o consumo sustentável. A associação positiva entre indivíduos das gerações mais velhas com o consumo sustentável de água contraria a expectativa teórica, em que se esperava que a geração mais nova fosse mais consciente para o consumo de água. Entretanto, tal resultado também foi encontrado no trabalho de Gilg e Barr (2006).

É oportuno destacar que em um contexto universitário, no qual os dados foram coletados, em maioria, quanto maior a idade, maior também o nível de escolaridade (alunos da graduação, mestrado, doutorado, professores). Nesse sentido, tal público pode ter influenciado os resultados.

A variável renda não apresentou correlação com a variável dependente. Contudo, optou-se por mantê-la, a fim de verificar se esta apresentaria influência no modelo de regressão, seguindo as orientações deNewell e Green (1997); Daciê, (2015) e Ribeiro e Veiga (2011).

Todas as variáveis demográficas foram testadas, o resumo do modelo está exposto na Tabela 8.

Tabela 8 - Regressão Logística

\begin{tabular}{|c|c|c|c|c|c|}
\hline Variáveis & $\underline{B}$ & $\underline{\text { S.E }}$ & $\underline{\text { Wald }}$ & $\underline{\text { Sig. }}$ & $\underline{\text { Exp. (B) }}$ \\
\hline Gênero(1) & $-0,496$ & 0,158 & 9,839 & 0,002 & 0,609 \\
\hline Escolaridade (2) & 0,584 & 0,279 & 4,392 & 0,036 & 1,794 \\
\hline Escolaridade (3) & 0,493 & 0,181 & 7,427 & 0,006 & 1,637 \\
\hline Renda (3) & 1,200 & 0,388 & 9,558 & 0,002 & 3,321 \\
\hline Renda (4) & 0,858 & 0,400 & 4,589 & 0,032 & 2,358 \\
\hline Constante & 20,829 & 40193,893 & 0,000 & 1,000 & \\
\hline
\end{tabular}

Fonte: Resultados da Pesquisa (2016)

As variáveis que compuseram o modelo, considerando o nível de significância à $5 \%$, foram Gênero, Escolaridade e Renda. Tal modelo vai ao encontro com as variáveis citadas pelos autores como influenciadoras do comportamento sustentável (STRAUGHAN e ROBERTS, 1999; STERN et al., 1999; DACIÊ et al., 2015; SANTOS e CONKE, 2014; VACCARI, 2014; RIBEIRO e VEIGA, 2011).

O R quadrado Cox \& Snell ajustado do modelo foi equivalente à 0,77 , e o valor do $\mathrm{R}$ quadrado Nagelkerke foi equivalente à 0,102 , todos os modelos tiveram baixo poder de explicação e a variação entre eles foi baixa, isto deve-se ao fato de que a escolha de um comportamento é uma variável complexa, podendo ser atribuída a diversos fatores além das variáveis demográficas (SOLOMON, 2011).

Analisando o valor de Wald, que conforme Hairet al. (2009) e Minussi, Damacena e Ness Jr. (2002) é medida mais indicada para se aferir o resultado da contribuição de variável independente para o modelo, pode-se afirmar que a variável gênero é a que mais contribui para o modelo, confirmando a expectativa teórica, que será explanada mais detalhadamente após o teste de médias. Além de ser possível constatar através da análise do Beta (B), que o gênero masculino é 0,496 vezes menos propenso ao comportamento sustentável. 


\section{(9REUNIR}

Sobre a escolaridade, o Beta permite-nos concluir que os respondentes com maior escolaridade estão mais propensos ao consumo sustentável de água. Este resultado está de acordo com a expectativa teórica, de que quanto mais uma população é estudada, mais ela é propensa ao comportamento sustentável (STRAUGHAN e ROBERTS, 1999; RIBEIRO e VEIGA, 2011).

Contrariando os resultados da correlação, a variável renda é significativa no modelo, demonstrando que quanto maior a renda, mais propício está o indivíduo ao consumo sustentável de água. Conforme o valor do Beta, os indivíduos que recebem de 10 a 12 salários mínimos estão 1,200 vezes mais propícios ao consumo sustentável, enquanto os indivíduos que recebem de 13 a 15 salários mínimos estão 0,858 vezes a este tipo de comportamento. Tais dados complementam os resultados da escolaridade, tendo em vista que, os indivíduos que recebem mais de 10 salários mínimos na população universitária são, em maioria, professores, possuindo maior escolaridade.

Os resultados da regressão demonstram que há relação das variáveis demográficas com o consumo sustentável da água, rejeitando-se a hipótese nula. Contudo, para complementar a análise, o teste de médias foi realizado para todas as variáveis, sendo rejeitada a hipótese nula de igualdade estatística de médias para todos os casos, com exceção da variável raça, resultado que coincide com a regressão. A intenção, contudo, deste teste de médias é verificar, quais, dentre as estratificações das variáveis apresentam maior média de consumo sustentável de água. Os testes de médias confirmaram os resultados da regressão.

Tabela 9 - Teste u de Mann Whitney - Gênero

\begin{tabular}{c|c|c}
\hline Gênero & Ranking de médias & $\underline{\text { Resumo do Ranking }}$ \\
\hline Feminino & 382,41 & 145697,50 \\
\hline Masculino & 334,74 & 113142,50 \\
\hline \multicolumn{2}{r}{ Fonte: Resultados da pesquisa (2016) }
\end{tabular}

Considerando o fato de que o teste de Mann-Whitney apresentou a rejeição da hipótese de igualdade estatística de médias entre os dois gêneros, confirma-se o pressuposto teórico de que o gênero feminino tende a ter maiores médias de comportamento sustentável, conforme apontam Straughan e Roberts (1999); Stern et al. (1999); Daciêet al. (2015); Santos e Conke (2014).

Com relação à escolaridade, a tabela 10 demonstra que o ranking de médias corresponde à expectativa teórica, em que os comportamentos sustentáveis estão mais associados com níveis mais altos de escolaridade, conforme apontam Straughan e Roberts (1999) e Ribeiro e Veiga (2011). A pós-graduação apresentou uma posição no ranking de médias superior aos demais níveis de escolaridade, o que é possível inferir que esse grupo está mais suscetível ao comportamento sustentável, particularmente o de água.

Tabela 10 - Teste u de Kruskal Wallis - Escolaridade

\begin{tabular}{l|c}
\multicolumn{1}{c|}{ Escolaridade } & Ranking de médias \\
\hline Ensino Fundamental & 320,75 \\
\hline Ensino Médio & 310,05 \\
\hline Ensino Superior & 331,59 \\
\hline Pós Graduação & 402,52 \\
\hline
\end{tabular}

Fonte: Resultados da pesquisa (2016) 
Quanto a variável renda, os dados demonstram que indivíduos de maior renda possuem maiores médias de comportamento sustentável em relação à água. Conforme demonstra a Tabela 11.

Tabela 11 - Teste u de Kruskal Wallis - Renda

\begin{tabular}{l|c}
\hline Renda & Ranking de médias \\
\hline 1 a 3 SM & 371,13 \\
\hline 4 a 6 SM & 369,58 \\
\hline 7 a 9 SM & 307,56 \\
\hline 10 a 12 SM & 323,82 \\
\hline 13 a 15 SM & 367,59 \\
\hline +15 & 429,79 \\
\hline \multicolumn{2}{|c}{ Fonte: Resultados da pesquisa (2016) }
\end{tabular}

A associação entre rendas mais elevadas com o consumo sustentável também foi constatada em outras pesquisas a exemplo de Berket al. (1993) e Gilg e Barr (2006), segundo esses autores, quanto maior a renda, mais inclinado está o indivíduo em adotar comportamentos que favorecem o consumo sustentável.

A partir dos resultados encontrados é possível verificar que os consumidores do gênero feminino, com maior nível de renda e escolaridade entre a apresentar comportamentos mais sustentáveis para o consumo de água. Entretanto, não foi verificada a relação entre a raça e o consumo sustentável, o que contraria dos resultados encontrados no estudo de De Oliver (1999), o qual foi constato que diferentes raças possuem diferentes comportamentos de consumo de água.Também não foi identificada a relação entre a idade dos indivíduos com o consumo sustentável, indo na contramão com a expectativa teórica.

\section{CONSIDERAÇÕES FINAIS}

A partir do desenho metodológico adotado, bem como do aparato teórico que guiou a elaboração desse estudo, foi possível atender ao objetivo proposto e compreender a relação entre as variáveis demográficas e o comportamento do consumidor de água. Ademais, a partir da validação do constructo consumo sustentável de água foi possível identificar a propensão dos respondentes ao uso consciente de água.

Os resultados da pesquisa permitem concluir o comportamento declarado do consumidor de água de Viçosa-MG,em média, épropício ao consumo sustentável. Ademais foi possível associar as variáveis demográficas em conformidade com a teoria, destaque para as variáveis gênero, escolaridade e renda. Tal fator permite-nos inferir que os públicos que apresentam menos propensão ao consumo sustentável da água merecem mais atenção, podendo ser público de campanhas ou estratégias que enfatizem a importância do consumo sustentável da água para a comunidade, principalmente em situações de restrição hídrica.

É relevante destacar, conforme já apontado por alguns autores que não há um conjunto de variáveis específicas para explicar determinado comportamento, sendo este, normalmente condicionados a diversos outros fatores, nem sempre mensuráveis. Desta forma, é interessante que futuras pesquisas se dediquem ao entendimento do comportamento do consumidor de água, relacionando-o com variáveis psicográficas e contextuais, tendo em vista que o conhecimento sobre o comportamento do consumidor se

68 


\section{(9)REUNIR}

faz bastante relevante para o incentivo da mudança ou permanência deste comportamento (KOLLMUS e ANGYEMAN, 2002; LEE E KOTLER, 2011).

Além disso, destaca-se a pertinência de pesquisas que incluam no escopo metodológico o uso de dados do comportamento real relacionado ao consumo de água, visto que nessa pesquisa fez-se uso apenas do comportamento declarado. Sugere-se a utilização das variáveis selecionadas para compor o construto comportamento sustentável de água, visto que o mesmo já obteve validação. Pode-se considerar a possibilidade de acréscimo de outras variáveis, a serem testadas, para a composição do constructo.

O contexto hídrico vivenciado pela região Sudeste fez com que o consumo de água fosse um tema debatido frequentemente pela sociedade como um todo, seja através da mídia ou da academia. Isto faz com que estudos como este possam contribuir para novas perspectivas em relação ao consumo de água e demais recursos naturais, cada vez mais escassos.

Deste modo, espera-se que a pesquisa tenha contribuído para chamar atenção ao tema do consumo de água e também apresentar indícios do perfil dos indivíduos mais propensos a economizar. $\mathrm{E}$, ainda, possa servir de convite para que demais pesquisadores estudem o comportamento do consumidor de água em outras populações, podendo enriquecer os estudos da área.

\section{AGRADECIMENTOS}

Agradecemos a Coordenação de Aperfeiçoamento de Pessoal de Nível Superior e a Fundação de Amparo à Pesquisa do Estado de Minas Gerais pelo financiamento da pesquisa.

\section{REFERÊNCIAS}

AFONSO, A. O consumidor verde:perfil e comportamento de compra.Dissertação. Programa de pós-graduação de Economia e Gestão. Universidade Técnica de Lisboa. Lisboa, 2010, p. 117.

AZEVÊDO, A.; CUNHA, F.; QUINELATO, R.; MADUREIRA, D.Consciência ambiental e comportamento do consumidor. In. SEMINÁRIOS DE ADMINISTRAÇÃO - SEMEAD, São Paulo, SP, Brasil. Anais... São Paulo/SP: Setembro de 2010, p.16.

BEDANTE, G. N. A influência da consciência ambiental e das atitudes com relação ao consumo sustentável na intenção de compras de produtos ecologicamente embalados. Dissertação. Programa de pós-graduação em Administração. Universidade Federal do Rio Grande do Sul. Porto Alegre-RS, 2004, p.159.

BERK, R.; SCHULMAN, D.; MCKEEVER, M.; FREEMAN, H. Measuring the impact of water conservation campaigns in California. Climatic Change, vol. 24, jul 1993, pp. 233-248.

BLACKWELL, R.; MINIARD, P.; ENGEL, J. Comportamento do consumidor.São Paulo, Pioneira Thomson Learning, 2005. 


\section{(9REUNIR}

BLAKE, J. Overcoming the 'value-action gap' in environmental policy: tensions between national policy and local experience. Local Environment, vol. 4, n. 3, 1999, pp. 257-278.

BRASIL.Constituição Federativa do Brasil. 1988. Disponível em: <http://www.planalto.gov.br/ccivil_03/Constituicao/Constituicao.htm>. Acesoem: 10 jun. 2017.

CAPELINI, M. Potencialidade e aplicação da prevenção de resíduos de embalagens: abordagem sobre o projeto do produto e o consumo. Tese de Doutorado. Programa de pósgraduação em Engenharia Ambiental. Universidade de São Paulo. São Paulo-SP, 2007. p. 257.

CUNHA, S.; COELHO, M. Política e gestão ambiental. In: CUNHA, S.; GUERRA, A. (Orgs). A questão ambiental: diferentes abordagens. Rio de Janeiro: Bertrand Brasil, 2003. pp. 43-76.

DACIÊ, F.; PANHOCA, L.; ESPEJO, M; BEZERRA, C. Quem é o "bicho verde"? Um estudo do comportamento verde de alunos do estado do Paraná. Organizações e Sustentabilidade, vol. 3, n. 1, jan/jun 2015, pp. 109-139.

DE OLIVER, M. Attitudes and Inaction: A Case Study of the Manifest Demographics of Urban Water Conservation. Environment and Behavior, vol. 31, n. 3, 1999, pp. 372-394.

FÁVERO, L.; SILVA, F.; BELFIORE, P.; CHAN, B. Análise de dados: Modelagem multivariada para tomada de decisões. Rio de Janeiro, Campus, 2009.

FISHBEIN, M.; AJZEN, I. Belief, Attitude, Intention, and Behavior: an introduction to theory and research.Reading, MA, Addison-Wesley Publications, 1975.

FRANCO, I. K. Valores e comportamento ecológico: Uma análise comparativa e evolutiva dos alunos de dois cursos de graduação da USP. Dissertação. Programa de pós-graduação em Administração. Universidade de São Paulo. Ribeirão Preto-SP, 2012, p.111.

G1. Racionamento de água já dura três meses em Viçosa. 19 Ago. 2016. Disponível em: <http://g1.globo.com/mg/zona-da-mata/noticia/2016/08/racionamento-de-agua-ja-duratres-meses-em-vicosa.html>. Acesso em: 02 ago. 2017.

GETZNER, M.; GRABNER-KRÄUTER, S. Consumer preferences and marketing strategies for "green shares" Specifics of the Austrian market. International Journal of Bank Marketing, vol.22, n.4, 2004, pp.260-278.

GILG, A; BARR, S. Behavioural attitudes towards water saving: Evidence from a study of environmental actions. Ecological Economics, vol.57, n.3, 2006, pp.400-414.

70 


\section{(9)REUNIR}

GOMES, G.; GORNI, P. M.; DREHER, M. Consumo sustentável e o comportamento de universitários: discurso e práxis. Revista Eletrônica de Ciência Administrativa, v. 10, n. 2, nov 2011, pp. 80-92.

GROHMANN, M.; BATTISTELLA, L.; VELTER, A; CASASOLA, F. Comportamento ecologicamente consciente do consumidor: adaptação da escala ECCB para o contexto brasileiro. Revista de Gestão Social e Ambiental, São Paulo, vol. 6, n.1, jan/abril 2012, pp.102-116.

HAIR, J.; ANDERSON, R.; TATHAM, R.; BLACK, W. Análise multivariada de dados. 6a ed. Porto Alegre, Bookman, 2009.

HINES, J.; HUNGERFORD, H.; TOMERA, A. Analysis and synthesis of research on responsible pro-environmental behavior: a meta-analysis. The Journal of Environmental Education, vol.18, n.2, 1986, pp.1-8.

KOLLMUSS, A.; AGYEMAN, J. Mind the Gap: why do people act environmentally and what are the barriers to pro-environmental behaviour? Environmental Educations Research, v.8, n.3, 2002, pp.239-260.

KOTLER, Philip; LEE, Nancy. Marketing Social: influenciando comportamentos para o bem. $3 \stackrel{a}{-}$ ed. Porto Alegre, Bookman, 2011.

LEONIDOU, L. C.; LEONIDOU, C. N.; KVASOVA, O. Antecedents and outcomes of consumer environmentally friendly attitudes and behaviour. Journal of Marketing Management, vol. 26, n.13, 2010, pp.1319-1344.

MAROCO, J.; GARCIA-MARQUES, T. Qual a confiabilidade do alfa de Cronbach? Questões antigas e soluções modernas. Laboratório de Psicologia, vol. 4, n.1, 2006, pp.65-90.

MINUSSI, J. A.; DAMACENA, C.; NESS JR, W. L. Um modelo de previsão de solvência utilizando regressão logística. Revista de Administração Contemporânea, v. 6, n. 3, p. 109128, 2002.

MISSIO, F.; JACOBI, L. Variáveis dummy: especificações de modelos com parâmetros variáveis. Ciência e Natura, vol. 29, n.1, 2007, pp.111-135.

NEWELL, S; GREEN, C. Racial Differences in Consumer Environmental Concern. Journal of Consumer Affairs, vol. 31, 1997, pp. 53-69.

RANDOLPH, B.; TROY, P. Attitudes to conservation and water consumption. Environmental science \& policy, vol. 11, n.5, 2008, pp. 441-455.

RIBEIRO, J.; VEIGA, R. Proposição de uma escala de consumo sustentável. Revista de Administração, vol.46, n.1, jan/fev/mar 2011, pp. 45-60.

71 


\section{QREUNIR}

ROBERTS, D. Landscape vegetation modelling with vital attributes and fuzzy systems theory. Ecological Modelling, vol. 90, n.2, 1996, pp. 175-184.

SAMARA, B.; MORSCH, M.Comportamento do consumidor: conceitos e casos. São Paulo, Pearson Prentice Hall, 2005.

SANTOS, M.; CONKE, L. Ciclistas e motoristas: consumidores ecologicamente corretos? Revista Eletrônica Gestão e Saúde. Edição Especial, 2014, pp.3397-14.

SOLOMON, M. R. O Comportamento do Consumidor:comprando, possuindo e sendo.9a ed. Porto Alegre, Bookman, 2011.

STERN, P.; TROY, T.; GUAGNANO, G.; KALOF, L. Society for Human EcologyA Value-BeliefNorm Theory of Support for Social Movements: The Case of Environmentalism Research in Human Ecology. Human Ecology Review, vol. 6, n.2, 1999, pp. 81-97.

STRAUGHAN, R.; ROBERTS, J. Environmental segmentation alternatives: a look at green consumer behaviour in the new millennium. Journal of Consumer Marketing, vol.16, n.6, 1999, pp. 558-575.

THOGERSEN, J.; SCHRADER, U. From knowledge to Action - New Paths Towards Sustainable Consumption. Journal of Consumer Policy, vol.35, n.1, 2012, pp.1-5.

TRIANDIS,H. Interpersonal behavior. Monterey,Brooks/Cole, 1977.

TRIOLA, M. F. Introdução à Estatística. 9 ed. Rio de Janeiro, LTC, 2005.

VACCARI, L. O hiato entre atitude e comportamento ecologicamente conscientes: Um estudo com consumidores de diferentes gerações. Tese de doutorado. Programa de pós-graduação em Administração. Pontifícia Universidade Católica do Rio de Janeiro. Rio de Janeiro-RJ, 2014, p.246.

WALL, R.; WRIGHT, P.; MILL, G. Organised Activity? A critical review of theoretical constructs used to explain environmentally-significant behaviour.Leicester, Mimeo, Montford University, 2004.

72 